\title{
Robust Adaptive Beamforming using k-means Clustering: A Solution to High Complexity of the Reconstruction-Based Algorithm
}

\author{
Zhihao TAN, Weimin JIA, Wei JIN \\ Dept. of Electronic \& Information Engineering, Xi'an Research Institute of High Technology, \\ Hongqing Town, Xi'an, 710025, People's Republic of China \\ tanzhihaoep@163.com,jwm602@163.com, jinweimail@126.com \\ Submitted July 13, 2017 / Accepted February 27, 2018
}

\begin{abstract}
Recently, a new robust adaptive beamforming $(R A B)$ algorithm has been proposed to reconstruct the interference-plus-noise covariance matrix (IPNCM) based on narrowing the interference angular domain and using an annular uncertainty set (NIAD-AUS). The method is robust against unknown arbitrary-type mismatches. However, its computational complexity will increase exponentially with the number of array sensors. In this paper, a novel method is proposed to solve this problem. First, $k$-means clustering (KMC) algorithm is utilized to estimate the annulus uncertainty set with fewer clustering weight points rather than whole sampling. Second, the KMC Capon spectrum is used to reconstruct the IPNCM. Compared with the previous reconstruction-based algorithms, the proposed approach can retain the high performance of the state-of-the-art NIAD-AUS algorithm. More importantly, it can also obtain the IPNCM more quickly. Lastly, simulation results demonstrate the effectiveness and robustness of the proposed algorithm.
\end{abstract}

\section{Keywords}

Robust adaptive beamforming, k-means clustering, reconstruction-based algorithm, low complexity

\section{Introduction}

The performances of traditional adaptive beamforming algorithms may suffer from imprecise knowledge of the desired signal steering vector [1]. In order to obtain a more accurate steering vector, some robust Capon beamforming algorithms were presented based on a smaller uncertainty set and iterative theory [6-8]. The performance of aforementioned algorithms is limited because they ignore the data-information excavation from the received data. In order to remove the desired signal components from the sample covariance matrix, a lot of RAB algorithms based on steering vector estimation and the IPNCM reconstruction have been proposed [9-14]. In [9], the IPNCM was reconstructed utilizing the Capon spectrum to the interference angular domain. To further reduce the computational complexity of the RAB method in [9], low-complexity interference-plus-noise covariance matrix reconstruction method based on spatial power spectrum sampling (SPSS) was proposed in [10] and [11]. To solve the problem of performance degradation with one single snapshot, a sparse signal recovery model under the non-convex optimization framework was first established in [12]. In [13], the iterative adaptive approach (IAA) algorithm is performed to acquire the true signal direction and the spatial spectrum simultaneously. To overcome the ineffectiveness in array calibration error, a more general algorithm [14] based on NIAD-AUS was proposed, and it can sufficiently collect more potential information using high-dimensional volume integral. In addition, it was proved that this algorithm [9] is a special category of the NIAD-AUS algorithm [14]. This method is robust against unknown arbitrary-type mismatches. However, a huge number of sample points for each angle are needed to obtain a more precise IPNCM, which results in the exponential increasing of CC.

In this paper, based on the previous reconstruction method [14], a modified one based on the $\mathrm{KMC}$ is proposed to reduce the CC efficiently. Different from the previous methods based on IPNCM reconstruction, our approach emphasizes the information excavation to sample points. The KMC approach can easily obtain clustering weight points to replace sample points in the annular uncertainty set, and it is essential to reduce the computational complexity of reconstructing the IPNCM. Subsequently, a more accurate steering vector of desired signal can be obtained by solving a quadratically constrained quadratic programming (QCQP) problem. The proposed beamformer can efficiently reduce the computation complexity with the negligible performance loss.

The rest of the paper is organized as follows. In Sec. 2, the problem formulation is given. Based on the IPNCM reconstruction and k-means clustering, a novel RAB algorithm is proposed in Sec. 3. In Sec. 4, the numerical simulations are presented to verify the performance. Finally, conclusion is drawn in Sec. 5. 


\section{Problem Formulation}

The purpose of robust adaptive beamforming is to find a common method which can steer the desired signal to the right direction and reduce the impact of the interferences and noise. It also means maximizing the output signal to interference plus noise ratio (SINR). Assume that a narrowband desired signal impinges on an array of $M$ sensors. The output of the beamformer is given by

$$
\boldsymbol{y}(k)=\boldsymbol{w}^{\mathrm{H}} \boldsymbol{x}(k)
$$

where $k$ is the time index, $\boldsymbol{w} \in C^{M \times 1}$ is the complex beamforming weight vector, $\boldsymbol{x}(k)=\left[x_{1}(k), \ldots, \mathrm{x}_{M}(k)\right]^{\mathrm{T}}$ is the complex vector of array observation, ()$^{\mathrm{T}}$ and ()$^{\mathrm{H}}$ stand for the transpose and Hermitian transpose operators, respectively. The observation vector is given by [2]

$$
\begin{aligned}
\boldsymbol{x}(k) & =\boldsymbol{s}(k)+\boldsymbol{i}(k)+\boldsymbol{n}(k) \\
& =s(k) \boldsymbol{a}+\boldsymbol{i}(k)+\boldsymbol{n}(k)
\end{aligned}
$$

where $\boldsymbol{s}(k), \boldsymbol{i}(k)$, and $\boldsymbol{n}(k)$ stand for the desired signal, interference, and noise components. The steering vector of the desired signal is given by

$$
\begin{aligned}
& \boldsymbol{a}\left(\theta_{0}\right)= \\
& {\left[1, \exp \left(-\mathrm{j} 2 \pi \frac{d}{\lambda} \sin \theta_{0}\right), \ldots, \exp \left(-\mathrm{j} 2 \pi \frac{d}{\lambda}(M-1) \sin \theta_{0}\right)\right]}
\end{aligned}
$$

where $\lambda$ is the wavelength, $d$ denotes the distance between the arrays, $\theta_{0}$ denotes the real direction of the desired signal. Hence, the received power can be obtained as

$$
P=\boldsymbol{w}^{\mathrm{H}} \boldsymbol{R} \boldsymbol{w}=\boldsymbol{w}^{\mathrm{H}} \boldsymbol{R}_{s} \boldsymbol{w}+\boldsymbol{w}^{\mathrm{H}} \boldsymbol{R}_{i+n} \boldsymbol{w}
$$

where $\boldsymbol{R}$ denotes the covariance matrix containing all components, $\boldsymbol{R}_{\mathrm{S}}$ and $\boldsymbol{R}_{\mathrm{i}+\mathrm{n}}$ denote the covariance matrix containing signal component and interference-plus-noise components, respectively. The output signal-to-interference-plusnoise ratio (SINR) can be formulated as

$$
S I N R=\frac{\boldsymbol{w}^{\mathrm{H}} \boldsymbol{R}_{\mathrm{s}} \boldsymbol{w}}{\boldsymbol{w}^{\mathrm{H}} \boldsymbol{R}_{\mathrm{i}+\mathrm{n}} \boldsymbol{w}} .
$$

Hence, the optimal weight vector can be obtained by maximizing the output SINR of the beamformer, namely, maximizing the power of the desired signal component and minimizing the power of interferences and noise components. The Capon beamformer assumes that the power of the desired signal is set as a fixed value, and the problem can be reformulated as [2]

$$
\min _{\boldsymbol{w}} \boldsymbol{w}^{\mathrm{H}} \boldsymbol{R}_{i+n} \boldsymbol{w} \quad \text { s.t. } \quad \boldsymbol{w}^{\mathrm{H}} \boldsymbol{a}\left(\theta_{0}\right)=1 .
$$

From (6), the following optimal weight vector can be found

$$
\boldsymbol{w}_{\mathrm{opt}}=\frac{\boldsymbol{R}_{\mathrm{i}+\mathrm{n}}^{-1} \boldsymbol{a}\left(\theta_{0}\right)}{\boldsymbol{a}^{\mathrm{H}}\left(\theta_{0}\right) \boldsymbol{R}_{\mathrm{i}+\mathrm{n}}^{-1} \boldsymbol{a}\left(\theta_{0}\right)} .
$$

In practical application, the estimated IPNCM is used rather than the exact IPNCM which is usually unavailable. In the previous algorithms, the sample covariance matrix stands for ideal IPNCM, which is based on maximum power distortionless response (MPDR) instead of maximum variance distortionless response (MVDR). MPDR is equal to MVDR when the estimated steering vector is accurate [15]. However, the beamformer performance will degrade seriously if the steering vector estimation is not accurate enough. Thus, MVDR is more suitable to be applied in RAB algorithms. According to the NIAD-AUS algorithm [14], the IPNCM $\hat{\boldsymbol{R}}_{\mathrm{i}+\mathrm{n}}$ can be reconstructed as

$$
\hat{\boldsymbol{R}}_{\mathrm{i}+\mathrm{n}}=\hat{\boldsymbol{R}}_{\mathrm{i}}+\hat{\boldsymbol{R}}_{\mathrm{n}}=\hat{\boldsymbol{R}}_{\mathrm{i}}+\sigma^{2} I
$$

where $\sigma^{2}$ is the noise power that can be approximately estimated by the minimum eigenvalue of sample covariance matrix $\hat{\boldsymbol{R}}$. We choose the Capon spectrum as an estimate of the spatial power spectrum over all possible direction.

$$
\hat{\sigma}_{\mathrm{i}}^{2}=\frac{1}{\boldsymbol{a}^{\mathrm{H}} \hat{\boldsymbol{R}}^{-1} \boldsymbol{a}} \quad(\theta \in \Theta)
$$

where $\boldsymbol{a}$ is the normal steering vector of the possible direction of the interference, $\Theta$ is the interference angular domain.

Thus, the interference covariance matrix can be calculated through

$$
\hat{\boldsymbol{R}}_{\mathrm{i}}=\frac{1}{2} \sum_{i=1}^{I} \sum_{l=1}^{L} \frac{\boldsymbol{a}_{i l}\left(\theta_{i}\right) \boldsymbol{a}_{i l}^{\mathrm{H}}\left(\theta_{i}\right)}{\boldsymbol{a}_{i l}^{\mathrm{H}}\left(\theta_{i}\right) \hat{\boldsymbol{R}}^{-1} \boldsymbol{a}_{i l}\left(\theta_{i}\right)}
$$

where $I$ is the number of sample points in $\Theta, L$ is the number of sample points on the surface of the each annulus uncertainty set.

\section{The Proposed KMC Method}

\subsection{Mismatch Model}

Considering that a proper mismatch model is crucial in improving the robustness of algorithm, the mismatch between the real steering vector $\boldsymbol{a}_{0}$ and the assumed steering vector $\overline{\boldsymbol{a}_{0}}$ is analyzed at first. In fact, mismatch is caused by various errors, such as look direction error, array calibration error, and so on. Fortunately, they all can be modeled as

$$
\boldsymbol{e}_{\mathrm{r}}=\left|\gamma_{\mathrm{r}}\right| \exp \left(\mathrm{j} \varphi_{\mathrm{r}}\right)
$$

where $\boldsymbol{e}_{\mathrm{r}}$ stands for a certain mismatch, including all types of errors, $\gamma_{\mathrm{r}}$ denotes the amplitude factor, and $\boldsymbol{\varphi}_{\mathrm{r}}$ is the phase vector, and those distribution functions are all unknown or indescribable.

Hence, the whole mismatch can be modeled to satisfy the following certain norm constraint 


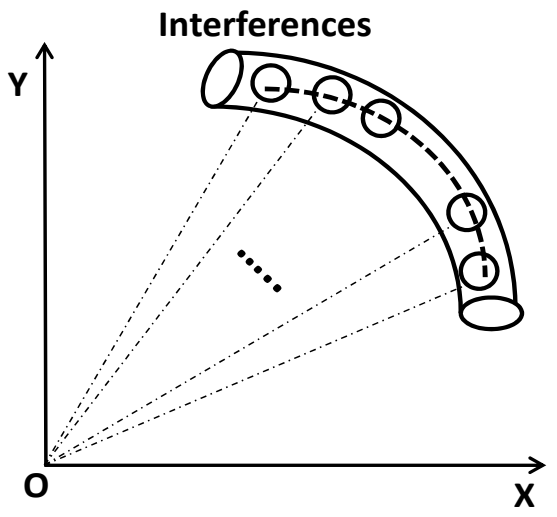

Fig. 1. The whole mismatch model based on uncertainty theory.

$$
\boldsymbol{e}=|\gamma| \exp (\mathrm{j} \boldsymbol{\varphi}) \quad, \quad\|\boldsymbol{e}\| \leq \varepsilon
$$

where $\boldsymbol{e}$ denotes the whole mismatch. Although the mismatch model may be inaccurate for a specific error, its performance is well against various errors. Hence, the whole mismatch model is meaningful for RAB algorithm. The real steering vector of the desired signal can be written as

$$
\boldsymbol{a}_{0}=\overline{\boldsymbol{a}}_{0}+\boldsymbol{e} .
$$

Figure 1 shows the whole mismatch model based on uncertainty theory. We assume that each steering vector error of the interferences can be covered by the uncertainty spherical set with the same size and all of them can be synthesized into a big annular uncertainty set. The steering vector located at the surface of the annulus can be constructed as [14]

$$
\boldsymbol{e}=\frac{\boldsymbol{\varepsilon}_{\mathrm{q}}}{\sqrt{M}}\left[\mathrm{e}^{\mathrm{j} \varphi_{0}}, \mathrm{e}^{\mathrm{j} \varphi_{1}}, \ldots, \mathrm{e}^{\mathrm{j} \varphi_{M-1}}\right]
$$

where $\varepsilon_{\mathrm{q}}$ denotes the norm of the random error vector, $\varphi_{m}(m=0,1 \ldots, M-1)$ are phases of the $m$ th coordinate of $\boldsymbol{e}$ and are uniformly distributed at intervals. The random error matrix can be written as

$$
\boldsymbol{E}=\left[\boldsymbol{e}_{1}, \boldsymbol{e}_{2}, \ldots, \boldsymbol{e}_{L}\right] .
$$

\subsection{KMC Algorithm}

The computational complexity of the NIAD-AUS algorithm is $o\left(L I M^{2}\right)$, which primarily comes from the IPNCM reconstruction. Thus, the number of sample points $L$ is essential in reducing computational complexity, while it increases exponentially with the number of the sensors.

$$
L=n_{0}^{M}\left(n_{0}=1,2,3 \ldots\right)
$$

where $n_{0}$ is a positive integer. Simulation results show that the performance barely improves when $n_{0} \geq 3$. So in this paper, we only research the $n_{0}=2$ case, which means that $\exp (\mathrm{j} \varphi)$ is 0 or 1 .

According to the previous analysis, the computational complexity of the NIAD-AUS is expressed as $o\left(2^{M} I M^{2}\right)$.

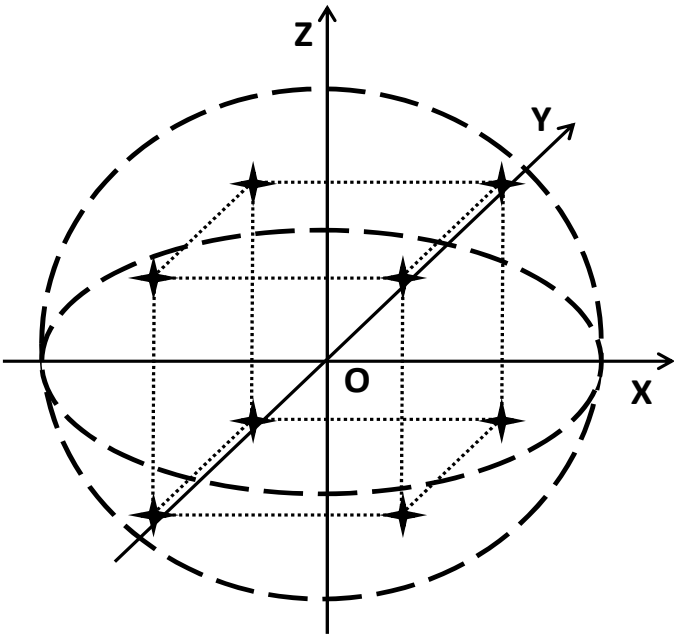

Fig. 2. The whole mismatch model in the 3-D space .

When the number of sensors grows, its computational complexity increases dramatically to reconstruct the IPNCM. As a result, it cannot meet the requirements of no or less time delay. Obviously, if the number of sample points decreases, the computational complexity will decrease while the performance will deteriorate. Thus, there is a contradiction between the low computational complexity and the outstanding performance.

Apparently, the uncertainty set is a convex hypersphere and the sample points are evenly distributed on its surface. To save performance loss, we select some weight points to replace the sample points.

Clustering can be considered as the most important unsupervised learning problem in data mining, which deals with finding a structure in the collection of unlabeled data. Thus, the clustering method can be used to obtain the weight points to resolve contradiction between the performance and the computational complexity. In this paper, we choose the k-means clustering method [16] for its simpleness and high efficiency. KMC aims to partition $n$ observations into $k$ cluster based on minimizing the within-cluster sum of squares (WCSS). The WCSS is the Euclidean distance. Thus, the objective function can be formulated as

$$
\arg \min _{\boldsymbol{S}_{=}=\left\{\boldsymbol{S}_{i}\right\}_{i=1}^{k}} \sum_{i=1}^{k} \sum_{\boldsymbol{e}_{l}=\boldsymbol{S}_{i}}\left\|\boldsymbol{e}_{l}-\boldsymbol{c}_{i}\right\|_{2}^{2}
$$

where $k$ is the number of cluster, $\boldsymbol{e}_{l}$ denotes the $l$ th sample point, $\boldsymbol{c}_{i}$ denotes the $i$ th cluster. Considering the structure of the sample points is obvious, one-iteration for $\mathrm{KMC}$ is enough to obtain the clustering points.

In general, it is difficult to calculate the whole mismatch model as a Capon spectrum integral over the uncertainty set. To solve the problem more easily, a method of using less discrete sample points to replace the whole uncertainty set is proposed in NIAD-AUS algorithm. Uncertainty set is only a structure to formulate the whole mismatch, and the sample points are chosen to describe the shape of structure. Figure 2 shows the whole mismatch model in the 3-D space. For the whole mismatch, those 
points distribute on the spherical surface, which can be formulated with the 8 spots of the cube. As we all know, only 4 noncoplanar points are needed to construct a sphere in general. Hence, the number of sample points can be reduced in theory. KMC effetively achieves the purpose of describing the whole mismatch with less weight points. Furthermore, it guarantees that the structure can describe the shape accurately.

\subsection{Steering Vector Estimation}

Using the same idea of [9], we can obtain a more precise steering vector by solving the following QCQP problem

$$
\begin{aligned}
& \min \quad\left(\overline{\boldsymbol{a}}_{0}+\boldsymbol{e}_{\perp}\right)^{\mathrm{H}} \hat{\boldsymbol{R}}^{-1}\left(\overline{\boldsymbol{a}}_{0}+\boldsymbol{e}_{\perp}\right) \\
& \text { s.t. } \quad \overline{\boldsymbol{a}}_{0}^{H} \boldsymbol{e}_{\perp}=0 \\
& \left(\overline{\boldsymbol{a}}_{0}+\boldsymbol{e}_{\perp}\right)^{\mathrm{H}} \hat{\boldsymbol{R}}_{\mathrm{i}+\mathrm{n}}\left(\overline{\boldsymbol{a}}_{0}+\boldsymbol{e}_{\perp}\right) \leq \overline{\boldsymbol{a}}_{0}^{H} \hat{\boldsymbol{R}}_{\mathrm{i}+\mathrm{n}} \overline{\boldsymbol{a}}_{0}
\end{aligned}
$$

where $\boldsymbol{e}_{\perp}$ is the orthogonal component of the mismatch vector $\boldsymbol{e}$. The objective function is the reciprocal of the outer power. The equality constraint is used to guarantee the orthogonality while the inequality constrain prevents the corrected steering vector from converging to any interference.

\subsection{Complexity Analysis}

In our algorithm, the main computational complexity lies in the IPNCM reconstruction, the KMC process, and the QCQP problem. The computational complexity of algorithms are listed in Tab. 1, where $S$ denotes the number of sample points in the complement of the angular domain, $t$ denotes the number of iterations, $o(t k L M)$ is the computational complexity of the KMC. It is worth emphasizing that $S>>M, L I>>M$, are assumed to guarantee the performance. Besides, the number of iteration is only once. The actual computational complexity can be obtained based on the above assumptions. The relationship between the complexities of the NIAD-AUS and the KMC can be described as computational complexity ratio (CCR).

$$
C C R=\frac{o(t k L M)}{o\left(L I M^{2}\right)}=\frac{k}{I M} .
$$

In general, $k$ and $M$ have the same order of magnitude. Thus, $C C R \approx 1 / I$, which means the proposed algorithm can significantly reduce the computational complexity compared with the NIAD-AUS.

\begin{tabular}{|c|c|c|}
\hline Algorithm & Theorical computational complexity & $\begin{array}{c}\text { Actual } \\
\text { computational } \\
\text { complexity }\end{array}$ \\
\hline $\begin{array}{c}\text { Algorithm } \\
{[9]}\end{array}$ & $\max \left(o\left(S M^{2}\right), o\left(M^{3.5}\right)\right)$ & $o\left(S M^{2}\right)$ \\
\hline $\begin{array}{c}\text { NIAD- } \\
\text { AUS }\end{array}$ & $\max \left(o\left(L I M^{2}\right), o\left(M^{3.5}\right)\right)$ & $o\left(L I M^{2}\right)$ \\
\hline Proposed & $\max \left(o\left(L I M^{2}\right), o\left(M^{3.5}\right), o(t k L M)\right)$ & $o(t k L M)$ \\
\hline
\end{tabular}

Tab. 1. Complexity analysis of algorithms.

\section{Numerical Analysis and Discussion}

A uniform linear array with half interelement spacing is employed. The number of isotropic sensors is fixed at 16 when considering the algorithm performance in terms of the input SNR. The true direction of the desired signal is $\theta_{0}=5^{\circ}$, and the assumed one is $\theta_{0}=2^{\circ}$. Two interferences, which have the same interference-to-noise ratio (INR) of $20 \mathrm{~dB}$, are impinging on the array from $\theta_{1}=-20^{\circ}$ and $\theta_{2}=-50^{\circ}$ off broadside to the array, respectively. The estimated direction of interferences are $\bar{\theta}_{1}=-23^{\circ}$ and $\bar{\theta}_{2}=-53^{\circ}$. The random error vector is drawn in each simulation run independently, $\varepsilon_{\mathrm{q}}$ is uniformly distributed in the interval $[0, \sqrt{0.3}]$ and $\varphi_{m}$ is uniformly distributed in the interval $[0,2 \pi)$. The number of sample points in $\Theta$, is assumed to be $I=40$. The number of snapshots is fixed at $K=30$. When comparing the performance in terms of the number of sensors, the signal-to-noise ratio (SNR) is set to be fixed at $0 \mathrm{~dB}$. In our simulations, we assume that the angular sector of the interferences is set to be $\left[\bar{\theta}_{1}-8, \bar{\theta}_{1}+8\right] \cup\left[\bar{\theta}_{2}-8, \bar{\theta}_{2}+8\right]$, which is the same as [10]. For each scenario in the simulations, 100 Monte Carlo runs are performed.

The performance of the proposed algorithm is compared with those of the robust Capon beamformer (RCB) [1], namely the iterative robust Capon beamformer (IRCB) [7], the reconstruction-based (RB) beamformer [9], and the NIAD-AUS beamformer [10]. We assume that the parameter $\varepsilon=9$ for RCB, the parameter $\varepsilon=0.08$ for IRCB, the

\begin{tabular}{|c|c|c|c|}
\hline $\boldsymbol{M}$ & NIAD-AUS $\left(\times \mathbf{1 0}^{\mathbf{9}}\right)$ & $\mathbf{K M C}\left(\times \mathbf{1 0}^{\mathbf{7}}\right)$ & $\mathbf{C C R}$ \\
\hline 10 & 0.004096000 & 0.0409600 & $10 \%$ \\
\hline 11 & 0.009912320 & 0.0901120 & $9.09 \%$ \\
\hline 12 & 0.023592960 & 0.1966080 & $8.33 \%$ \\
\hline 13 & 0.055377920 & 0.4259840 & $7.69 \%$ \\
\hline 14 & 0.128450560 & 0.9175040 & $7.14 \%$ \\
\hline 15 & 0.294912000 & 1.9660800 & $6.67 \%$ \\
\hline 16 & 0.671088640 & 4.1946040 & $6.25 \%$ \\
\hline 17 & 1.515192320 & 8.9128960 & $5.88 \%$ \\
\hline 18 & 3.397386240 & 18.8743680 & $5.56 \%$ \\
\hline 19 & 7.570718720 & 39.8458880 & $5.26 \%$ \\
\hline 20 & 16.77721600 & 83.8860800 & $5.00 \%$ \\
\hline
\end{tabular}

Tab. 2. Complexities versus number of sensors.

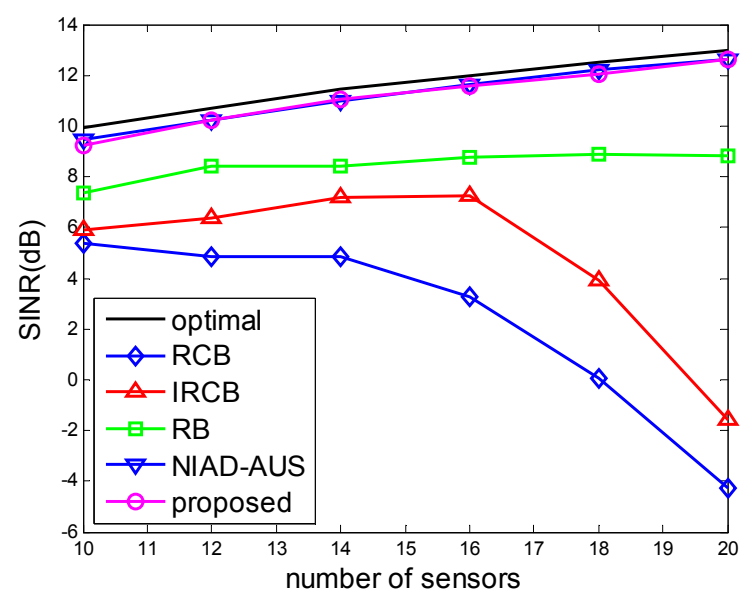

Fig. 3. Output SINRs versus number of sensors. 


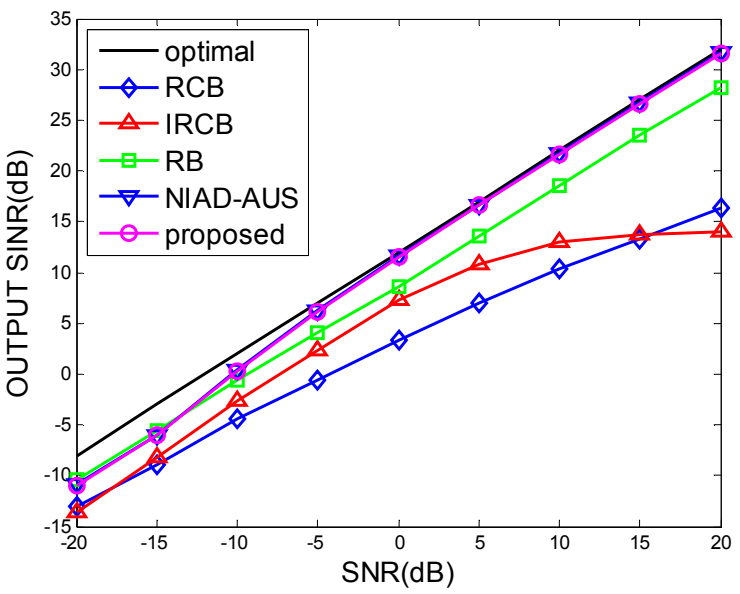

Fig. 4. Output SINRs versus the input SNRs.

parameter $S=1024$ for $\mathrm{RB}$, and the parameter $\varepsilon=\sqrt{0.1}$ for the NIAD-AUS algorithm and the proposed algorithm, the number of clumsers $k=40$. Convex optimization toolbox, CVX, is used to solve these optimization problems.

In the first example, we set $\mathrm{SNR}=0 \mathrm{~dB}$ and focus on studying the effect of the number of array sensors. Table 2 shows the computational complexity of the beamformers and Figure 3 presents the performance of output SINR. In Tab. 2 the advantage in computational complexity is more prominent when the number of sensors increases. The computational complexity of the proposed algorithm can reduce to $5 \%$ of the previous NIAD-AUS. The significant reduction in computational complexity is a direct result of using the less clustering points to replace the whole sample points. From Fig. 3, it is easy to find out that the performances of the RCB and the IRCB decrease in varying degrees. This is due to the direct adoption of sample covariance matrix without information excavation. The other three reconstruction-based algorithms show outstanding performances even if $M$ becomes larger, and the loss between the NIAD-AUS and the proposed algorithm is negligible.

In the second example, let SNRs vary from $-20 \mathrm{~dB}$ to $20 \mathrm{~dB}$, the number of sensors is fixed at 16. The SINRs are investigated in Fig. 4. According to the figures and tables, it is clearly drawn that the computational complexity of the proposed KMC algorithm is much less than that of the NIAD-AUS when their performance is very close. The maximum loss of SINR performance is approximately $0.2 \mathrm{~dB}$.

Figure 5. shows the output SINRs versus the number of snapshots when $\mathrm{SNR}=0 \mathrm{~dB}$ is set. As we can see from the figure, the RCB and the IRCB converge slowly and approximately need 100 snapshots to acquire the maximum of algorithms. The proposed algorithm converges quickly, and can keep high performance even if the number of snapshots is 10. Actually, the reconstruction-based algorithm can reduce the demand for snapshots because of full use of $\hat{\boldsymbol{R}}$.

In order to further examine the performance of the algorithm, we also analyze the relationship between the

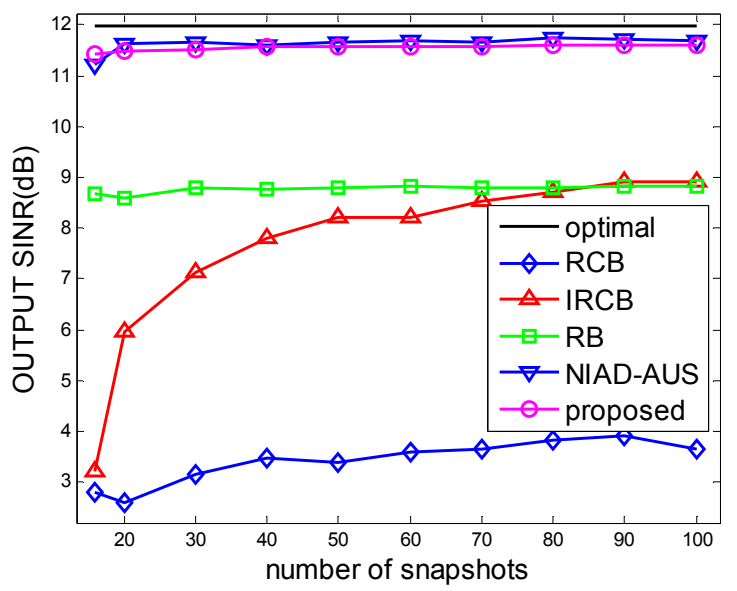

Fig. 5. Output SINRs versus number of snapshots.

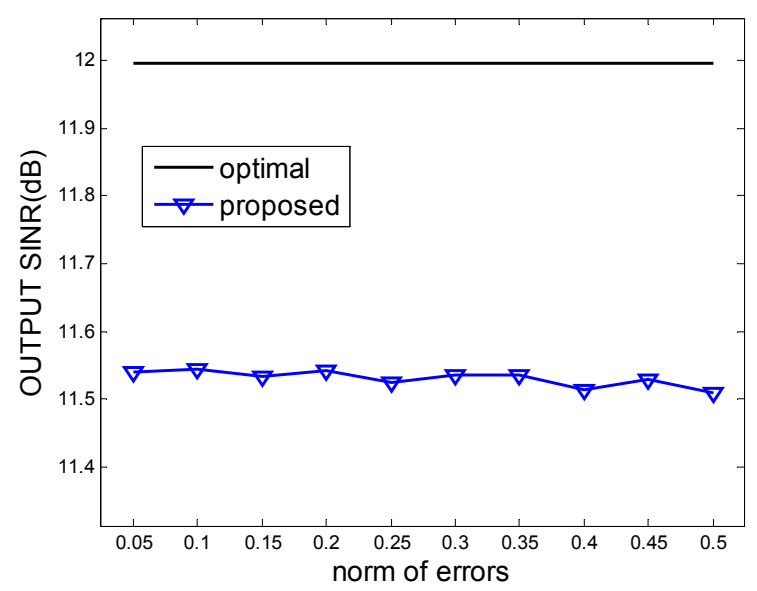

Fig. 6. Output SINRs versus norm of errors $\boldsymbol{\varepsilon}_{\mathrm{q}}$.

norm of errors $\varepsilon_{\mathrm{q}}$ and the output SINR. In Fig. 6, we change the errors distribution parameter from 0.05 to 0.50 . It is notable that the uncertainty set parameter $\varepsilon$ is $\sqrt{0.1}$, which is less than 0.50 . It is obviously that the performance can maintain the outstanding performance in the whole field, even when the number of uncertainty set parameter is a little bit less than the real errors set parameter. Thus, the proposed algorithm has strong robustness for its insensitivity to parameter.

We have verified the assumptions in the previous analysis of the number of iterations and weight sample points in the third example. Figure 7 shows the output SINRs versus the number of iterations. It is easy to find that one-iteration is enough to acquire the structure to describe the whole mismatch model. Figure 8 describes the output SINRs versus the number of weight sample points. The NIAD-AUS needs $2^{M}$ sample points for reconstruction, approximately ranging 1024 1048576. However, 40 weight points are enough to obtain the similar performance for the proposed algorithm. The KMC is used to describe the structure to formulate the whole mismatch. This structure is used to accurately describe all sampling points. However, it is still not possible to include all information. Thus, there's a loss of performance. Fortunately, the performance loss is negligible. 


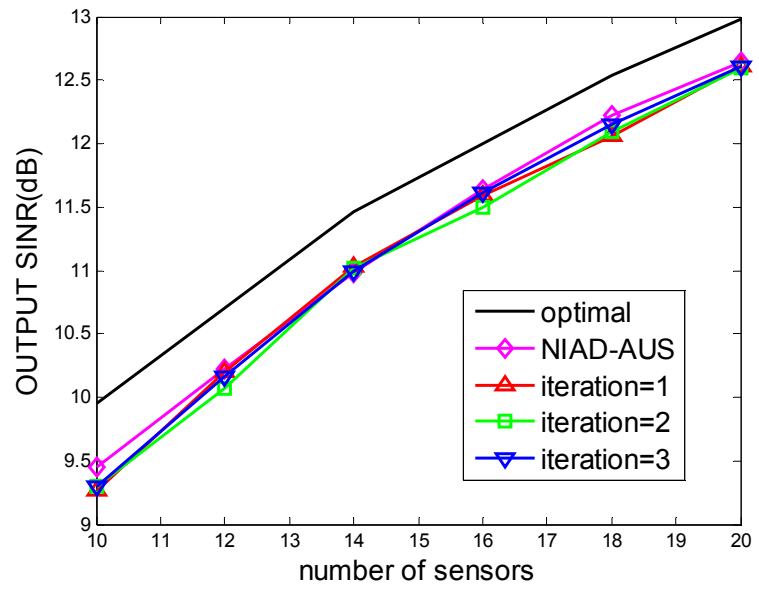

Fig. 7. Output SINRs versus number of iterations.

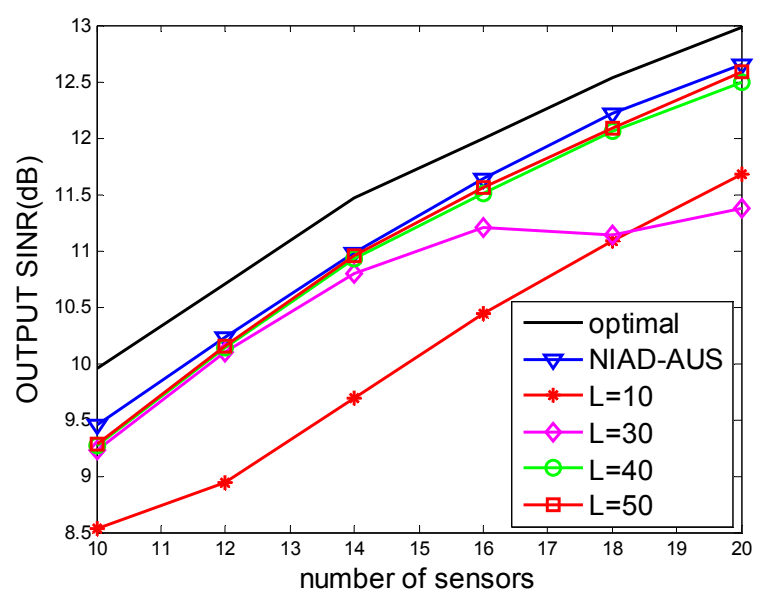

Fig. 8. Output SINRs versus number of the weight sample points.

\section{Conclusions}

A new IPNCM reconstruction algorithm based on kmeans clustering for robust adaptive beamforming is proposed. The proposed algorithm can easily obtain clustering weight points to reduce the high computational complexity of the IPNCM reconstruction. Compared with the previous NIAD-AUS algorithm, the computational complexity can be reduced by 1-2 orders of magnitude while the loss of performance is negligible. Simulation results have been presented to support the effectiveness and robustness of the proposed algorithm.

\section{Acknowledgments}

This work was supported by the National Nature Science Foundation of China under grants 61179004 and 61179005 .

\section{References}

[1] LI, J., STOICA, P., WANG, Z. On robust Capon beamforming and diagonal loading. IEEE Transactions on Signal Processing, 2003, vol. 51, no. 7, p. 1702-1715. DOI: 10.1109/ICASSP.2003.1199947

[2] VOROBYVO, S. A., GERSHMAN, A. B., LUO, Z. Q. Robust adaptive beamforming using worst-case performance optimization: a solution to the signal mismatch problem. IEEE Transactions on Signal Processing, 2003, vol. 51, no. 2, p. 313-324. DOI: 10.1109/TSP.2002.806865

[3] LI, J., STOICA, P., WANG, Z. Doubly constrained robust Capon beamformer. IEEE Transactions on Signal Processing, 2004, vol. 52, no. 9, p. 2407-2423. DOI: 10.1109/TSP.2004.831998

[4] DU, L., LI, J., STOICA, P. Fully automatic computation of diagonal loading levels for robust adaptive beamforming. IEEE Transaction on Aerospace and Electronic Systems, 2010, vol. 46, no. 1, p. 449-458. DOI: 10.1109/TAES.2010.5417174

[5] JIA, W., JIN, W., ZHOU, S, et al. Robust adaptive beamforming based on a new steering vector estimation algorithm. Signal Processing, 2013, vol. 93, no. 9, p. 2539-2542. DOI: 10.1016/j.sigpro.2013.03.015

[6] NAI, S. E., SER, W., YU, Z. L., et al. Iterative robust minimum variance beamforming. IEEE Transactions on Signal Processing, 2011, vol. 59, no. 4, p. 1601-1611. DOI: 10.1109/TSP.2010.2096222

[7] JIN, W., JIA, W., YAO, M., et al. Iterative doubly constrained robust Capon beamformer. Electronics Letters, 2011, vol. 47, no. 25 , p. $1372-1373$. DOI: $10.1049 /$ el.2011.3360

[8] JIN, W., JIA, W., YAO, M., et al. Robust adaptive beamforming based on iterative implementation of worst-case performance optimization. Electronics Letters, 2012, vol. 48, no. 22, p. 1389-1391. DOI: 10.1049/el.2012.1718

[9] GU, Y., LESHEM, A. Robust adaptive beamforming based on interference covariance matrix reconstruction and steering vector estimation. IEEE Transactions on Signal Processing, 2012, vol. 60, no. 7, p. 3881-3885. DOI: 10.1109/TSP.2012.2194289

[10] GU, Y., GOODMAN, N. A., HONG, S., et al. Robust adaptive beamforming based on interference covariance matrix sparse reconstruction. Signal Processing, 2014, vol. 96, no. 5, p. 375-381. DOI: $10.1016 /$ j.sigpro.2013.10.009

[11] ZHANG, Z., LIU, W., LENG, W., et al. Interference-plus-noise covariance matrix reconstruction via spatial power spectrum sample for robust adaptive beamforming. IEEE Signal Processing Letters, 2016, vol. 23, no. 1, p. 121-125. DOI 10.1109/LSP.2015.2504954

[12] CHEN, P., ZHAO, Y., LIU, C. Robust adaptive beamforming based on sparse reconstruction using non-convex optimization algorithm. Electronics Letters, 2016, vol. 52, no. 19, p. 1584-1586. DOI: $10.1049 / \mathrm{el} .2016 .1810$

[13] WANG, Y., BAO, Q., CHEN, Z. Robust adaptive beamforming using IAA-based interference-plus-noise covariance matrix reconstruction. Electronics Letters, 2016, vol. 52, no. 13, p. 1185-1186. DOI: 10.1049/el.2015.4420

[14] HUANG, L., ZHANG, J., XU, X. Robust adaptive beamforming with a novel interference-plus-noise covariance matrix reconstruction method. IEEE Transactions on Signal Processing, 2015, vol. 63, no. 7, p. 1643-1650. DOI: 10.1109/TSP.2015.2396002 
[15] VAN TREES, H. L. Optimum Array Processing. $1^{\text {st }}$ ed., rev. New York (USA): Wiley-Interscience, 2002. ISBN: 9780471093909

[16] LLOYD, S. P. Least squares quantization in PCM. IEEE Transactions on Information Theory, 1982, vol. 28, no. 2, p. 129-137. DOI: 10.1109/TIT.1982.1056489

\section{About the Authors ...}

Zhihao TAN was born in 1992. He is studying for his M.Sc. from Xi'an Research Inst. of High Technology, China. His research interests include the array signal processing and robust adaptive beamforming.
Weimin JIA was born in Hebei, China in 1971. She received her Ph.D. degree from Xi'an Research Ins. of High Technology, Xi'an, China in 2007. She is a professor in the Dept. of Communication Engineering, Xi' an Research Inst. of High Technology. Her current research interests include the Sat-COM and smart antennas.

Wei JIN was born in Hubei, China in 1984. He received his Ph.D. degree from Xi'an Research Inst. of High Technology, Xi'an, China in 2013. He is a lecturer in the Dept. of Communication Engineering, Xi' an Research Inst. of High Technology. His current research interests include the array signal processing and radar signal processing. 